Article

\title{
On the Modelling of the Transient Flow Behavior of Metallic Glasses: Analogy with Portevin-Le Chatelier Effect
}

\author{
Nashmi Hassan Alrasheedi ${ }^{1}$, Mohamed Abdelbasset Yousfi ${ }^{2}$, Khalil Hajlaoui ${ }^{1,2, *}$, \\ Bacem Jacem Mahfoudh ${ }^{2}$, Zoubeir Tourki ${ }^{2}$ and Alain Reza Yavari ${ }^{3,+}$ \\ 1 Al Imam Mohammad Ibn Saud Islamic University (IMSIU), Othman Ibn Affan Street, \\ Riyadh 11432, Saudi Arabia; nashminashmi68@gmail.com \\ 2 Laboratoire de Mécanique de Sousse (LMS), ENISo, Technopole de Sousse, University of Sousse, \\ BP 254 Erriadh 4023, Tunisia; yousfiyousfi744@yahoo.fr (M.A.Y.); bjmahfouth@gmail.com (B.J.M.); \\ tourkiz89@gmail.com (Z.T.) \\ 3 SIMaP-LTPCM, Institut National Polytechnique de Grenoble, 38402 Saint Martin d'Heres, France \\ * Correspondence: khalil.hajlaoui@eniso.rnu.tn; Tel.: +216-73-369-500; Fax: +216-73-369-506 \\ $+\quad$ This author deceased on 24 November 2015.
}

Academic Editor: Hugo F. Lopez

Received: 4 December 2015; Accepted: 25 February 2016; Published: 29 February 2016

\begin{abstract}
A constitutive model for serrated flow together with a finite element (FE) analysis was developed to simulate discontinuous yielding behavior of metallic glasses, commonly known as serrated flow inhomogeneous deformation. The constructed model is based on transient behavior resulting from the time dependence of the flow defect concentration in the shear bands. The computed results of stress-strain curves were consistent with the experimental data. The main features of the serrated flow effect have also been exhibited by the model.
\end{abstract}

Keywords: metallic glasses; serrated flow; flow defects; shear bands

\section{Introduction}

At room temperature, Bulk Metallic Glasses (BMGs) typically deform inhomogeneously with plastic flow highly localized into nanoscale regions, namely shear bands [1-3]. The outstanding consequence of shear banding is the serrated flow event which is characterized in the stress-strain curves by repeated cycles of a sudden stress drops followed by an elastic reloading. Despite extensive studies, the physical origin of serrated flow and its connection with the shear banding process is still under advanced examination. There exists, however, a general consensus that the shear band initiation is caused by local structural softening owing to a free volume generation [4-6]. The serrated flow was also recognized to occur by stick-slip operation of a single shear band, as has been reported recently [7-9]. While a great effort has been made to understand and detail the physical causes of shear bands initiation and propagation, it remains an open question about the mechanism that governs the shear-band arrest (Why do shear bands stop?). Several intrinsic and extrinsic factors have been suggested to cause the arrest of the shear banding operation. We have experimentally established that the in situ nanocrystallization within the shear bands can offer a "self-locking effect" and increases the strength of shear regions preventing further propagation of shear bands [10-12]. Few authors have considered a type of relaxation mechanism able to increase the resistance to the shear bands motion [13]. The diverse approaches for shear band propagation and arrest have recognized the key role of free volume $[6,14,15]$ as well as the effect of temperature and strain rate. 
The serrated flow events are not unique to BMGs. In certain crystalline alloys, they can be observed in distinct temperature and strain rate ranges and it is usually known as the Portevin-Le Chatelier (PLC) effect [16] where the dynamics stick-slip arise from the collective pinning and unpinning of dislocations from solute atmosphere, commonly known as the dynamic strain aging (DSA) mechanism [17].

Furthermore, strong similarities in the deformation behavior of amorphous metals with that of crystalline alloys (exhibiting Portevin-Le Chatelier bands) were reviewed in many previous works $[7,13]$. These phenomenological similarities are seen mainly in the occurrence of serrated flow and the negative strain rate sensitivity which may stem from a similar micromechanical origin. In view of these similarities, we adopt a new approach to analyze the serrated flow of BMGs in an effort to obtain insight into the complex and irregular form of stress-strain curves (i.e., serrations). This approach, therefore, comes from the phenomenological similarities found between the serrated flow in amorphous metal and the DSA effect in crystalline materials.

In this work, a macroscopic constitutive model is presented that includes a time-varying state variable which accounts for the local degree of relaxation in the shear band after a shear event has taken place. The model is based on the transient behavior of shear banding resulting from the time dependence of the flow defect concentration, $C_{\mathrm{f}}$. The transition from high to low $C_{\mathrm{f}}$ in a shear zone can be described by the "relaxation-saturation" process as described by Dalla Torre et al. [7,18]. The proposed elasto-viscoplastic model has been implemented in a finite element program and the non-linear equations have been integrated using a Runge-Kutta method. Computer simulations of the flow serration were carried out and the effect of strain rates was numerically studied.

\section{Constitutive Model for Serrated Flow}

\subsection{Micromechanism of Serrated Flow}

The fluctuations in the stress-strain curves during plastic deformation of certain crystalline metallic alloys (known as the PLC effect [16]) have been attributed to the collective pinning and unpinning of dislocations from solute atmosphere [19], giving rise to a stick and slip phases, respectively (known as the dynamic strain ageing (DSA) mechanism [20]). Then, the constitutive models employed for the DSA mechanism have included the local solute concentration $C_{\mathrm{s}}$ at arrested mobile dislocations as a time dependent state variable.

On the other hand, in spite of the similarities with the BMGs and the associated inhomogeneous deformation [21], the DSA model obviously cannot be applicable to amorphous metals because it operates with dislocation-based mechanisms. Its framework, however, may provide a basis for refining the constitutive models of plastic deformation in metallic glasses where it operates with the concept of structural defects. Indeed, during straining of BMGs, shear dilatation processes were believed to increase the free volume content $[22,23]$ and destroy the local short-range order within the shear bands. This suggests that the state variable could be the content of a free volume (or local concentration of the flow defects $C_{f}$ ) within the shear band, which gradually decreases the viscosity and induces structural softening.

Following Torre et al. [7], the shear bands propagation in metallic glasses is controlled by the interplay between the cooperative shear motion of atomic clusters (Shear Transformation Zones (STZs)) and some unknown relaxation mechanisms promoted by diffusion and local atomic rearrangements, increasing the resistance to further shear propagation. The latter mechanism should be responsible for the hardening and the arrest of the shear banding. The extent of the structural relaxation may counteract the local increase of the free volume (or $C_{\mathrm{f}}$ ), such that the net free volume within the shear band after relaxation is lower than the one at the moment of shearing, which leads to a higher structural density. This leads to the strengthening of the distorted structure and it can compensate for the local softening, preventing the runaway failure along the shear band. Consequently, the shear process stops and the material turns into elastic form until the next shear event (serration) takes place (as experimentally shown in Figure 1a and schematically illustrated in Figure 1b). 

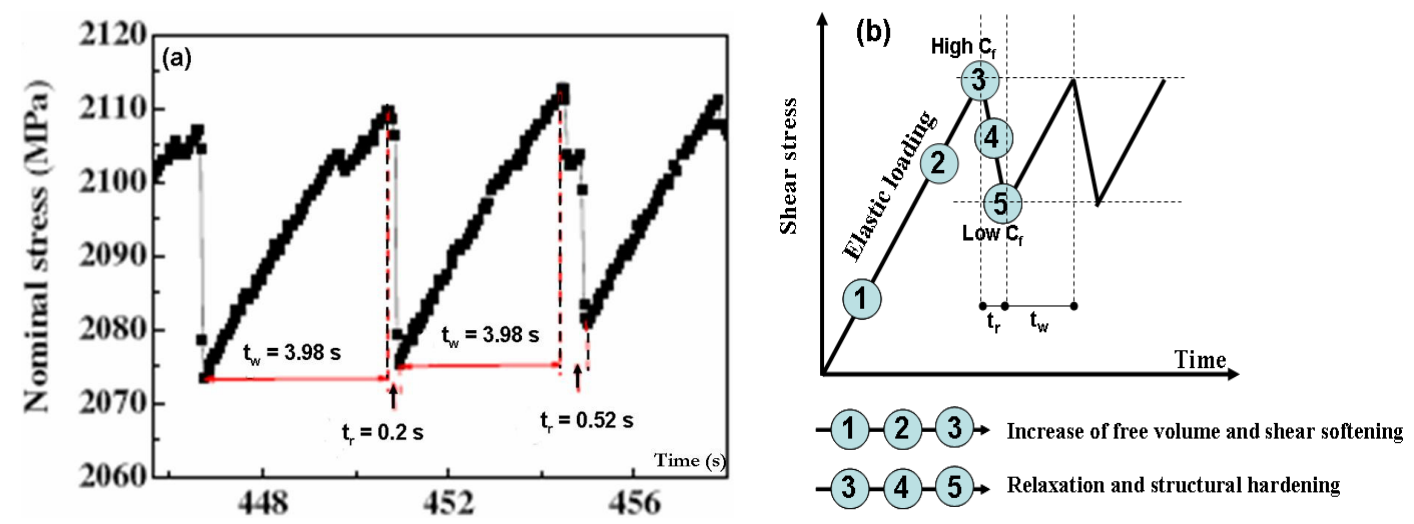

Figure 1. (a) Experimental stress-time curve of deformed Zr-based Bulk Metallic Glasses (BMG) showing typical serrations in time scale [24]; (b) The corresponding schematic illustration of successive serration events. $C_{\mathrm{f}}$ is the flow defect concentration (free volume content), $t_{\mathrm{r}}$ is the relaxation time (the stress drop time) and $t_{\mathrm{w}}$ is the waiting time (the stress increasing time).

Therefore, the structural state variable $C_{\mathrm{f}}$ accounts for the degree of structural relaxation after a shear event and hence governs the temperature and the strain rate dependence of the serrated flow deformation characteristics. The constitutive model of dynamic serrated flow phenomena must include $C_{\mathrm{f}}$ as a time depending variable. For conditions where the atomic diffusion and reordering is operating, $C_{\mathrm{f}}$ is a dependent function of the waiting time $\left(t_{\mathrm{w}}\right)$ between two successive serration events. The extent of structural relaxation, however, is limited by the relaxation time $t_{\mathrm{r}}$ (as illustrated in Figure $1 \mathrm{~b}$ ).

\subsection{Constitutive Equations and FE Implementation}

Previous analysis has shown that the shear softening and the relaxation-induced hardening in metallic glasses are kinetically and thermally activated mechanisms. The total deformation is the sum of elastic and plastic strains tensors and the constitutive model for serrated flow can be introduced as follows:

$$
\widetilde{\varepsilon}=\widetilde{\varepsilon}^{\mathrm{e}}+\widetilde{\varepsilon}^{\mathrm{p}}
$$

Furthermore, it has been argued that the yielding of metallic glasses could rather be described as obeying to the Drucker Prager criterion rather than the von Mises criterion. The yield function can be defined as follows:

$$
f(\underline{\sigma})=(1-\lambda) \cdot J_{2}(\underline{\sigma})+\lambda \sigma_{\text {moy }}-R_{0}-R(\gamma)-R_{\mathrm{r}}\left(\gamma, t_{\mathrm{r}}\right)
$$

where $J_{2}(\underline{\sigma})$ is the second invariant of the stress tensor, $\lambda$ is a material constant, $\sigma_{\text {moy }}$ is the mean stress, $R_{0}$ is the yield stress in the absence of structural relaxation, $R(\gamma)$ is the isotropic nonlinear hardening law and $R_{\mathrm{r}}\left(\gamma, t_{\mathrm{r}}\right)$ is the extra-hardening induced by structural relaxation mechanism as described below. For metallic glasses, the stress-strain curve consists generally of an elasticity followed by a yielding and a perfect plasticity. Then, strain hardening is absent and $R(\gamma)$ can be neglected. $\gamma$ is the cumulated plastic flow.

The shear-relaxation hardening is assumed to be isotropic and it includes the new state variables, i.e., local concentration of the flow defects $C_{\mathrm{f}}$ :

$$
R_{\mathrm{r}}\left(\gamma, t_{\mathrm{r}}\right)=P_{1} \cdot C_{\mathrm{f}}
$$

The free volume concentration, $C_{\mathrm{f}}$, within the shear bands is estimated as a function of the cumulated plastic strain, $\gamma$, and the extent of relaxation time, $t_{\mathrm{r}}$.

$$
C_{\mathrm{f}}=\exp \left(-P_{2} \cdot \gamma^{m} \cdot t_{\mathrm{r}}{ }^{n}\right)
$$


where $P_{1}, P_{2}, m$ and $n$ are material model parameters. $P_{2}$ (unit: $\cdot \mathrm{s}^{-n}$ ) characterizes the rate of decreasing of free volume during the shear banding and $P_{1}$ (unit: $\mathrm{MPa}$ ) characterizes the intensity in stress of the resultant relaxation effect.

$C_{\mathrm{f}}$ is between 1 and 0 and decreases with increasing relaxation time $t_{\mathrm{r}}$. A situation of shear initiation and structural softening corresponds to $C_{\mathrm{f}} \approx 1$, a situation of shear arrest results from a low flow defect concentration i.e., $C_{\mathrm{f}} \approx 0$. The transition from high to low $C_{\mathrm{f}}$ (which occurs during shear banding) corresponds to the "relaxation-hardening" kinetics (Steps (3) $\rightarrow$ (4) $\rightarrow$ (5) in Figure 1b).

The cumulated plastic flow rate is deduced from the normality law [5]:

$$
\dot{\gamma}=\alpha_{0} \cdot v_{0} \cdot \gamma_{0} \cdot \exp \left(-\frac{Q_{\mathrm{STZ}}}{K_{\mathrm{B}} \cdot T}\right) \sinh \left(\frac{f(\underline{\sigma}) \cdot V_{\mathrm{a}}}{\sqrt{3} K_{\mathrm{B}} \cdot T}\right)
$$

and:

$$
\dot{\tilde{\varepsilon}}^{P}=\dot{\gamma} \frac{\partial f}{\partial \widetilde{\sigma}}
$$

where $\alpha_{0}$ is a constant incorporating the fraction of material that is available to undergo a shear transformation zone, $v_{0}$ is a characteristic attempt frequency and $\gamma_{0}$ is the characteristic strain of an STZ event. $Q_{\text {stz }}$ is the free energy for the activation of shear transformation, given by Equation (7) [5]:

$$
Q_{S T Z}=\left[\frac{7-5 v}{30 \cdot(1-v)}+\frac{2 \cdot(1+v)}{9 \cdot(1-v)} \beta^{2}+\frac{1}{2 \cdot \gamma_{0}} \cdot \frac{\tau_{0}}{\mu(T)}\right] \cdot \mu(T) \cdot \gamma_{0}^{2} \cdot \Omega_{0}
$$

where $v$ is the Poisson ratio, $\tau_{0}$ is the stress to initiate the shear event and $\mu(T)$ is the temperature-dependent shear modulus. $\Omega_{0}$ is the volume of an STZ sustaining a shear strain $\gamma_{0}$. $\beta$ is a parameter about equal to unity for metallic glasses. $V_{\mathrm{a}}$ is the apparent activation volume, expressed by [7]:

$$
V_{\mathrm{a}}=K_{\mathrm{B}} \cdot T \cdot \frac{\Delta \ln (\dot{\gamma})}{\Delta \sigma}
$$

Here $K_{\mathrm{B}}$ and $T$ are the Boltzmann constant and the temperature, respectively. The time dependence of the diffusion mediated atomic-arrangement within shear bands can be described by the "relaxation-saturation" kinetics of Avrami and expressed similar to as suggested in reference [25] by:

$$
\dot{t}_{\mathrm{r}}=\frac{t_{\mathrm{w}}-t_{\mathrm{r}}}{t_{\mathrm{w}}} ; t_{\mathrm{w}}=\frac{w}{\dot{\gamma}}
$$

$t_{\mathrm{r}}$ refers to time-duration of the diffusive atomic rearrangement in shear bands. The waiting time $t_{\mathrm{w}}$ is defined as the time between two successive serrations. $w$ is the plastic strain increment produced by shear event.

The formulated Equations (1)-(9) provide a closed elastic-viscoplastic constitutive model for serrated flow in deformed metallic glasses. These non-linear equations are solved using an implicit Newton-Raphson integration technique in which the corresponding material tangent is obtained by simultaneously and implicitly integrating the plastic flow and the flow defect equations. The specimens (plate of $12.5 \mathrm{~mm} \times 2.5 \mathrm{~mm}$ dimensions) used in the simulations are 8-node quadratic elements with reduced integration under plane stress conditions. Simulations have been performed at a constant strain rate of $10^{-3} \mathrm{~s}^{-1}$.

\section{Simulation Results}

\subsection{Stress-Displacement Curve}

The stress-displacement curve, obtained from simulations of uniaxial compressive tests is fitted to experimental stress-displacement data. The numerical result is compared to the experimental deformation tests of Liu et al. [26] where their results revealed an elastic deformation followed by 
a clearly jerky type deformation with transient shear banding events registered with displacement bursts in the stress-strain curve of $\mathrm{Zr}_{55} \mathrm{Pd}_{10} \mathrm{Cu}_{20} \mathrm{Ni}_{5} \mathrm{Al}_{10}$ glassy rods.

Figure 2 shows the experimental stress-displacement curve of the deformed Zr-based glassy alloy together with the best simulated stress-displacement curve obtained on the basis of equations presented in Section 2.2 using the parameter values listed in Table 1.

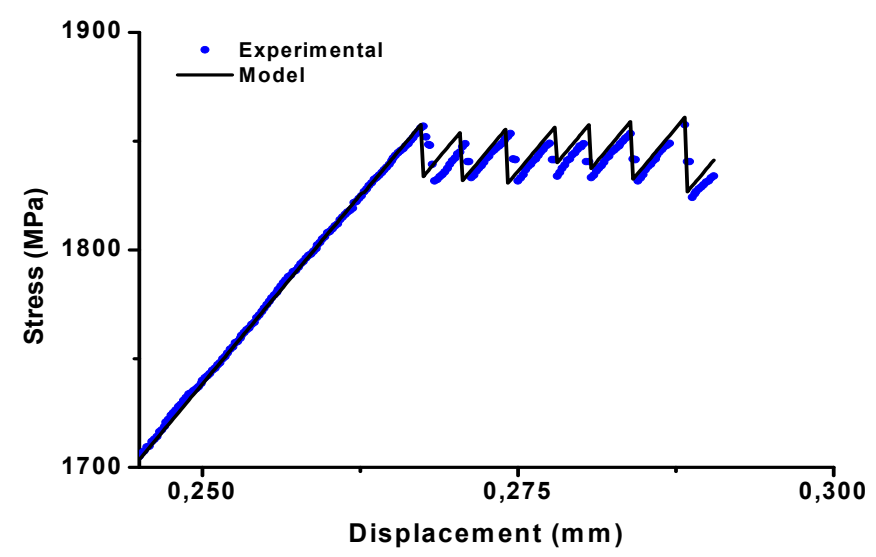

Figure 2. Stress-displacement curves of the deformed Zr-based glassy alloy. The line curve is the result obtained from the numerical simulation.

Table 1. Fitting parameters used for the simulation of the serrated flow behavior in BMGs alloys. Results from literature were also presented for comparison.

\begin{tabular}{ccc}
\hline Parameters & Values & Values from Literature \\
\hline$E(\mathrm{GPa})$ & 106.830 & $90-115[27]$ \\
$\lambda$ & 0.18 & - \\
$P_{1}(\mathrm{MPa})$ & 20.36 & $18[25]$ \\
$P_{2}\left(\mathrm{~s}^{-n}\right)$ & 4.06 & $3.91[28]$ \\
$v_{0}\left(\mathrm{~s}^{-1}\right)$ & $9.78 \times 10^{7}$ & - \\
$w$ & $9.65 \times 10^{-5}$ & $10^{-4}[28]$ \\
$Q_{\mathrm{STZ}}(\mathrm{J})$ & $1.01 \times 10^{-19}$ & $0.1 \mathrm{eV}[29]$ \\
$V_{\mathrm{a}}\left(\mathrm{m}^{3}\right)$ & $2.28 \times 10^{-28}$ & $0.15 \times 10^{-27}-4 \times 10^{-27}[30]$ \\
$\gamma_{0}$ & 0.1 & $0.1[5]$ \\
$v$ & 0.2 & $0.2-0.3[31]$ \\
$\alpha_{0}$ & 1 & - \\
$m$ & 1 & $0.44[28]$ \\
$n$ & 0.32 & $0.33[28]$ \\
\hline
\end{tabular}

From the simulation results presented in Figure 2, it can be concluded that the simulation fits quite well to the experimental results. After a certain amount of plastic strain, slight serrations were found on the macroscopic stress-displacement curve. Almost of the adjustable model parameters obtained are physically meaningful and comparable with those found in the literature. $Q_{\mathrm{STZ}}, V_{\mathrm{a}}$ and $E$ are close to that obtained in references $[5,27,29,30]$. The value of $\gamma_{0}, v_{0}, \alpha_{0}$ is in agreement with similar published results [32]. The Values of $m, n, P_{1}, P_{2}$ and $w$ are comparable to those found in References [25,28].

\subsection{Strain Rate Effects}

Using the model parameters in Table 1, simulations of compressive tests were carried out for various strain rates. Figure 3 shows the simulated stress-strain behavior of BMG with alternating the applied strain rate between $5 \times 10^{-5} \mathrm{~s}^{-1}$ and $1 \times 10^{-4} \mathrm{~s}^{-1}$. 


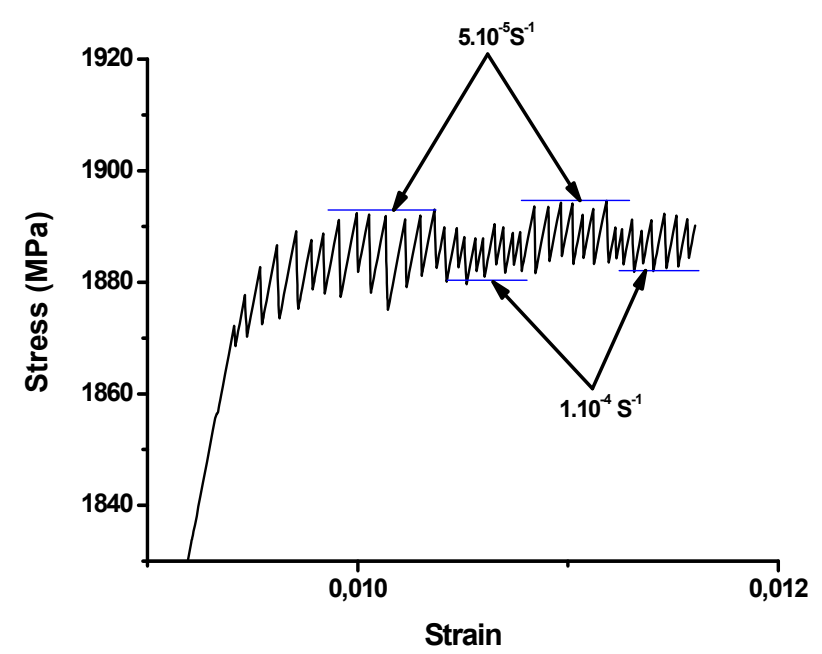

Figure 3. Simulated deformation curve of BMG alloy at oscillating strain rates of $5 \times 10^{-5} \mathrm{~s}^{-1}$ and $1 \times 10^{-4} \mathrm{~s}^{-1}$ (at room temperature) using the parameters of Table 1 .

The flow stresses showed higher values at lower strain rates, giving rise to negative strain rate sensitivity which is a required condition for serrated flow deformation in a range of BMGs alloys to occur $[33,34]$. From the obtained results, we suggest that, if the time for the structural relaxation (diffusive free volume annihilation) within the shear bands is sufficient after a shear displacement, the yield strength is higher, but it decreases for shorter periods of time, i.e., for higher applied strain rates. In other words, according to the physical picture of our model, when the applied strain rate suddenly decreases, more time is available for the relaxation to occur between successive stress drops. This time allows for the sheared zone to annihilate more free volume. In turn, this leads to a strengthening of the shear deformed zone, or macroscopically to a higher stress resistance against flow than reached by a rapidly applied strain rate. Therefore, a higher flow stress results at lower applied strain rates. This causes a negative strain rate sensitivity which is commonly known for metallic glasses showing serrated flow (see Figure 1 in reference [34] for comparison) as well as for crystalline materials exhibiting PLC effect.

\section{Conclusions}

1. A new constitutive model for the discontinuous yielding in metallic glasses was proposed. It includes the local flow defect concentration within the arrested shear bands as a time dependent state variable. The simulated model showed an agreement with the experimental data for the serrated flow behavior in BMGs as well as the main related features, i.e., strain rate effect.

2. Despite the essential difference between deformation in crystalline and amorphous metals, the above model based on the comparison of deformation in BMGs and the DSA mechanism suggests that the phenomenological similarities found for both classes of metals may stem from a similar micromechanical origin.

Acknowledgments: The authors are very glad to dedicate this paper to the memory of Alain Reza Yavari (one of the authors) who passed away recently.

Author Contributions: K.H. and N.H.A. constructed the constitutive model, wrote and edited the paper and contributed to numerical implementation. M.A.Y. and B.J.M. contributed to the numerical simulations. Z.T. and A.R.Y. contributed to the discussion and interpretation of the results.

Conflicts of Interest: The authors declare no conflict of interest. 


\section{References}

1. Schuh, C.A.; Hufnagel, T.C.; Ramamurty, U. Mechanical behavior of amorphous alloys. Acta Mater. 2007, 55, 4067-4109. [CrossRef]

2. Trexler, M.M.; Thadhani, N.N. Mechanical properties of bulk metallic glasses. Prog. Mater. Sci. 2010, 55, 759-839. [CrossRef]

3. Chen, M.W. Mechanical Behavior of Metallic Glasses: Microscopic Understanding of Strength and Ductility. Annu. Rev. Mater. Res. 2008, 38, 445-469. [CrossRef]

4. Spaepen, F. A microscopic mechanism for steady state inhomogeneous flow in metallic glasses. Acta Metall. 1977, 25, 407-415. [CrossRef]

5. Argon, A.S. Plastic deformation in metallic glasses. Acta Metall. 1979, 27, 47-58. [CrossRef]

6. Falk, M.L.; Langer, J.S. Dynamics of viscoplastic deformation in amorphous solids. Phys. Rev. E 1998, 57, 7192-7205. [CrossRef]

7. Torre, F.H.D.; Dubach, A.; Schallibaum, J.; Loffler, J.F. Shear striations and deformation kinetics in highly deformed Zr-based bulk metallic glasses. Acta Mater. 2008, 56, 4635-4646. [CrossRef]

8. Song, S.X.; Bei, H.; Wadsworth, J.; Nieh, T.G. Flow serration in a Zr-based bulk metallic glass in compression at low strain rates. Intermetallics 2008, 16, 813-818. [CrossRef]

9. Maßß, R.; Klaumunzer, D.; Loffler, J. Propagation dynamics of individual shear bands during inhomogeneous flow in a Zr-based bulk metallic glass. Acta Mater. 2011, 59, 3205-3213. [CrossRef]

10. Hajlaoui, K.; Yavari, A.R.; Doisneau, B.; LeMoulec, A. Shear delocalization and crack blunting of a metallic glass containing nanoparticles: In situ deformation in TEM analysis. Scri. Mater. 2006, 54, 1829-1834. [CrossRef]

11. Hajlaoui, K.; Yavari, A.R.; LeMoulec, A.; Botta, W.J. Plasticity induced by nanoparticle dispersions in bulk metallic glasses. J. Non-Crys. Sol. 2007, 353, 327-331. [CrossRef]

12. Hajlaoui, K.; Yavari, A.R.; Das, J.; Vaughan, G. Ductilization of BMGs by optimization of nanoparticle dispersion. J. Alloy. Compd. 2007, 434-435, 6-9. [CrossRef]

13. Dubach, A.; Torre, F.H.D.; Loffler, J.F. Deformation kinetics in Zr-based bulk metallic glasses and its dependence on temperature and strain rate sensitivity. Phil. Mag. Lett. 2007, 87, 695-704. [CrossRef]

14. Greer, A.L.; Cheng, Y.Q.; Ma, E. Shear bands in metallic glasses. Mater. Sci. Eng. R 2013, 74, 71-132. [CrossRef]

15. Langer, J.S. Microstructural shear localization in plastic deformation of amorphous solids. Phys. Rev. E 2001, 64, 011504. [CrossRef] [PubMed]

16. Le Chatelier, F. Influence du temps et de la température sur les essais au choc. Rev. Metall. 1909, 6, 914-917.

17. Chihab, K.; Estrin, Y.; Kubin, L.P.; Vergnol, J. The kinetics of the Portevin-Le Chatelier bands in Al-5 at. \% Mg Alloy. J. Scr. Metall. 1987, 21, 203-208. [CrossRef]

18. Torre, F.H.D.; Klaumunzer, D.; Maaß, R.; Loffler, J.F. Stick-slip behavior of serrated flow during inhomogeneous deformation of bulk metallic glasses. Acta Mater. 2010, 58, 3742-3750. [CrossRef]

19. Ananthakrishna, G.; Bharathi, M.S. Dynamical approach to the spatiotemporal aspects of the Portevin-Le Chatelier effect: Chaos, turbulence, and band propagation. Phys. Rev. E 2004, 70, 026111. [CrossRef] [PubMed]

20. Mulford, R.A.; Kocks, U.F. New observations on the mechanism of dynamic strain aging and jerky flow. Acta Metall. 1979, 27, 1125-1134. [CrossRef]

21. Sarmah, R.; Ananthakrishna, G.; Sun, B.A.; Wang, W.H. Hidden order in serrated flow of metallic glasses. Acta Mater. 2011, 59, 4482-4493. [CrossRef]

22. Hajlaoui, K.; Benameur, T.; Vaughan, G.; Yavari, A.R. Thermal expansion and indentation-induced free volume in $\mathrm{Zr}$-based metallic glasses measured by real-time diffraction using synchrotron radiation. Scri. Mater. 2004, 51, 843-848. [CrossRef]

23. Flores, K.M.; Kanungo, B.P.; Glade, S.C.; Asoka-Kumar, P. Characterization of plasticity-induced structural changes in a Zr-based bulk metallic glass using positron annihilation spectroscopy. J. Non-Cryst. Sol. 2007, 353, 1201-1207. [CrossRef]

24. Wang, G.; Chan, K.C.; Xia, L.; Yu, P.; Shen, J.; Wang, W.H. Self-organized intermittent plastic flow in bulk metallic glasses. Acta Mater. 2009, 57, 6146-6155. [CrossRef] 
25. Zhang, S.; McCormick, P.G.; Estrin, Y. The morphology of Portevin-Le Chatelier bands: Finite element simulation for Al-Mg-Si. Acta Mater. 2000, 49, 1087-1094. [CrossRef]

26. Liu, Y.H.; Liu, C.T.; Gali, A.; Inoue, A.; Chen, M.W. Evolution of shear bands and its correlation with mechanical response of a ductile $\mathrm{Zr}_{55} \mathrm{Pd}_{10} \mathrm{Cu}_{20} \mathrm{Ni}_{5} \mathrm{Al}_{10}$ bulk metallic glass. Intermetallics 2010, 18, 1455-1464. [CrossRef]

27. Louzguine-Luzgin, D.V.; Louzguina-Luzgina, L.V.; Churyumov, A.Y. Mechanical Properties and Deformation Behavior of Bulk Metallic Glasses. Metals 2013, 3, 1-22. [CrossRef]

28. Graff, S.; Forest, S.; Strudel, J.-L.; Prioul, C.; Pilvin, P.; Bechade, J.-L. Finite Element Simulations of Dynamic Strain Ageing Effects at V-Notches and Crack Tips. Scr. Mater. 2005, 52, 1181-1186. [CrossRef]

29. Li, L.; Homer, E.R.; Schuh, C.A. Shear transformation zone dynamics model for metallic glasses incorporating free volume as a state variable. Acta Mater. 2013, 61, 3347-3359. [CrossRef]

30. Torre, F.H.D.; Dubach, A.; Loffler, J.F. Deformation kinetics of Zr-based bulk metallic glasses-Temperature and strain rate influences on shear banding. J. Alloys. Compd. 2010, 495, 341-344. [CrossRef]

31. Yu, P.; Bai, H.Y. Poisson's ratio and plasticity in CuZrAl bulk metallic glasses. Mater. Sci. Eng. A 2008, 485, 1-4. [CrossRef]

32. Argon, A.S.; Kuo, H.Y. Free energy spectra for inelastic deformation of five metallic glass alloys. J. Non-Cryst. Solids 1980, 37, 241-266. [CrossRef]

33. Torre, F.H.D.; Dubach, A.; Nelson, A.; Loffler, J.F. Temperature, strain and strain rate dependence of serrated flow in bulk metallic glasses. Mater. Trans. 2007, 48, 1774-1780. [CrossRef]

34. Torre, F.H.D.; Dubach, A.; Siegrist, M.E.; Loffler, J.F. Negative strain rate sensitivity in bulk metallic glass and its similarities with the dynamic strain aging effect during deformation. Appl. Phys. Lett. 2006, 89, 091918. [CrossRef]

(C) 2016 by the authors; licensee MDPI, Basel, Switzerland. This article is an open access article distributed under the terms and conditions of the Creative Commons by Attribution (CC-BY) license (http:/ / creativecommons.org/licenses/by/4.0/). 